UCRL-JC-126325

PREPRINT

\title{
High Yield Cu-Co CPP GMR Multilayer Sensors
}

\author{
J. Spallas, M. Mao, B. Law, \\ F. Grabner, C. Cerjan, and D. O'Kane
}

This paper was prepared for submittal to the

International Magnetics Conference 1997

New Orleans, Louisiana

April 1-4, 1997

January 15, 1997

This is a preprint of a paper intended for publication in a journal or proceedings. Since changes may be made before publication, this preprint is made available with the understanding that it will not be cited or reproduced without the permission of the author. 


\section{DISCLAIMER}

This document was prepared as an account of work sponsored by an agency of the United States Government. Neither the United States Government nor the University of California nor any of their employees, makes any warranty, express or implied, or assumes any legal liability or responsibility for the accuracy, completeness, or usefulness of any information, apparatus, product, or process

disclosed, or represents that its use would not infringe privately owned rights. Reference herein to any specific commercial product, process, or service by trade name, trademark, manufacturer, or otherwise, does not necessarily constitute or imply its endorsement, recommendation, or favoring by the United States Government or the University of California. The views and opinions of authors expressed herein do not necessarily state or reflect those of the United States Government or the University of California, and shall not be used for advertising or product endorsement purposes. 


\title{
Improved Performance of Cu-Co CPP GMR Multilayer Sensors
}

\author{
J. P. Spallas, M. Mao, B. Law, F. Grabner, D. O'Kane ${ }^{\text {a) }}$, and C. Cerjan \\ Lawrence Livermore National Laboratory, Livermore, CA 94550

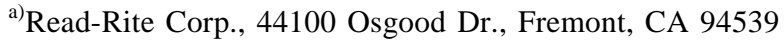

\begin{abstract}
We have fabricated and tested GMR magnetic flux sensors that operate in the CPP mode. This work is a continuation of the ultra-high density magnetic sensor research introduced at INTERMAG 96. We have made two significant modifications to the process sequence. First, contact to the sensor is made through a metal conduit deposited in situ with the multilayers. This deposition replaces electroplating. This configuration ensures a good electrical interface between the top of multilayer stack and the top contact, and a continuous, conductive current path to the sensor. The consequences of this modification are an increase in yield of operational devices to $\geq 90 \%$ per wafer and a significant reduction of the device resistance to $\leq 560 \mathrm{~m} \Omega$ and of the uniformity of the device resistance to $\leq 3 \%$. Second, the as-deposited multilayer structure has

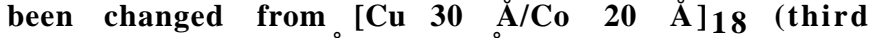
peak) to $\left[\begin{array}{llllll}\mathrm{Cu} & 20.5 & \mathrm{~A} / \mathrm{Co} & 12 & \AA\end{array}\right] 30$ (second peak) to increase the CPP and CIP responses. The sheet film second peak CIP GMR response is $18 \%$ and the sensitivity is $0.08 \% /$ e. The sheet film third peak CIP GMR response is $8 \%$ and the sensitivity is 0.05 \%/Oe. The second peak CPP GMR response averaged over twenty devices on a four inch silicon substrate is $28 \% \pm 6 \%$. The response decreases radially from the substrate center. The average response at the center of the substrate is $33 \% \pm 4 \%$. The average second peak CPP sensitivity is $0.09 \% / 0$ e \pm 0.02 \%/Oe. The best second peak CPP response from a single device is $39 \%$. The sensitivity of that device is $0.13 \% / O e$. The third peak CPP GMR response is approximately $14 \%$. The third peak CPP response sensitivity is $0.07 \% / O e$.
\end{abstract}

\section{INTRODUCTION}

This research is part of a larger effort to develop a biased, shielded magnet flux sensor that can be scaled to perform at densities $>25$ Gbits/in2 [1]. The magnetic sensor is composed of a giant magnetoresistive (GMR) multilayer (ML) film and is designed to operate in the current perpendicular to the plane (CPP) mode. The advantages to using the CPP mode are: current flow in the direction perpendicular to the ML film plane gives a greater GMR response than current flow in the ML plane [2], and output voltage $(\Delta \mathrm{V})$ will increase as the sensor diameter is decreased. Therefore, as the diameter of a CPP mode sensor is decreased to improve the spatial resolution, the output signal should increase. It is the focus

Manuscript received January 31, 1997.

J. P. Spallas, e-mail spallas1@1lnl.gov, phone 510-423-5304, FAX 510422-8761.

This work was performed under the auspices of the U.S. Department of Energy by the Lawrence Livermore National Laboratory under contract number W-7405-Eng-48 and supported in part under advanced magnetic heads cooperative research and development agreement number TC-84094. 
of this research to fabricate submicron diameter test devices. The goal is to demonstrate that a GMR ML sensor configured for CPP mode operation capable of multi-Gbits/in ${ }^{2}$ performance will have a sufficiently large $\Delta \mathrm{V}$ and sensitivity to be practical for magnetic information retrieval.

The test device is composed of a GMR ML sensor that is patterned using electron beam lithography. The ML is deposited in situ onto the bottom contact. The top contact is self-aligned to the sensor. This is accomplished, in part, by chemical mechanical polishing (CMP). The top and bottom contacts are electrically isolated by a plasma enhanced chemical vapor deposited (PECVD) $\mathrm{Si}_{3} \mathrm{~N}_{4}$ film. The configuration of the contacts allows four point probe resistance measurements. The fabrication details and the quasistatic test (QST) results for test devices fabricated using $\mathrm{Cu}$ Co third peak ML spacing have been published [3]. The third peak test devices can be characterized by the following: (1) The ML structure was [Cu $30 \AA /$ Co $20 \AA]$ 18. (2) The sensor diameter was nominally $0.43 \mu \mathrm{m}$. (3) The device resistance was typically many ohms varied by as much as $13 \%$ per wafer. (4) The yield of operational devices was typically less than $20 \%$ per wafer. (5) The sensitivity was $\leq 0.1 \% / O e$.

We have addressed the low yield, low sensitivity, and high device resistance by making two significant modifications to the fabrication sequence. First, contact to the sensor is made through a metal conduit deposited in situ with the multilayers. This is done in place of the electroplating processes described in [3]. This configuration ensures a good interface between the top of ML stack and the top contact, and a continuous, conductive current path to the sensor. Second, the as-deposited ML structure has been changed to $[\mathrm{Cu} 20.5$ $\AA /$ Co $12 \AA]_{30}$ (second peak) to increase the CPP and CIP responses. This paper will focus on the $\mathrm{Cu}-\mathrm{Co}$ second peak ML spacing CPP GMR test devices. We will outline the processes developed to fabricate CPP GMR devices detailing the process modifications. We will present device resistance, QST, and yield data for these test structures. Finally, we will discuss how performance of these devices can be further improved.

\section{FABRICATION}

The test devices are fabricated on four inch diameter (100) silicon substrates. The bottom contact is a Mo-Si ML deposited by low pressure dc magnetron sputtering. By optimizing the deposition parameters, $>0.5 \mu \mathrm{m}$ thick Mo-Si multilayers can be deposited with resistivity $<100 \mu \Omega$-cm and $<0.2 \mathrm{~nm}$ RMS roughness [4]. The nominal structure is

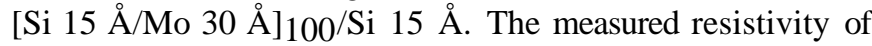
the Mo-Si ML bottom contact is approximately $25 \mu \Omega-\mathrm{cm}$. This Mo-Si ML is continuous and exhibits no amplification of roughness or columnar growth throughout the stack [4]. The $\mathrm{Cu}-\mathrm{Co} \mathrm{ML}$ is deposited onto the Mo-Si ML without breaking vacuum. The nominal structure is $[\mathrm{Cu} 20.5 \AA / \mathrm{Co}$ $12 \AA]_{30}$. The thickness uniformity of the $\mathrm{Cu}-\mathrm{Co} \mathrm{ML}$ is better than $1 \%$ over a four inch substrate [5]. A $500 \mathrm{~nm} \mathrm{Cu}$ 


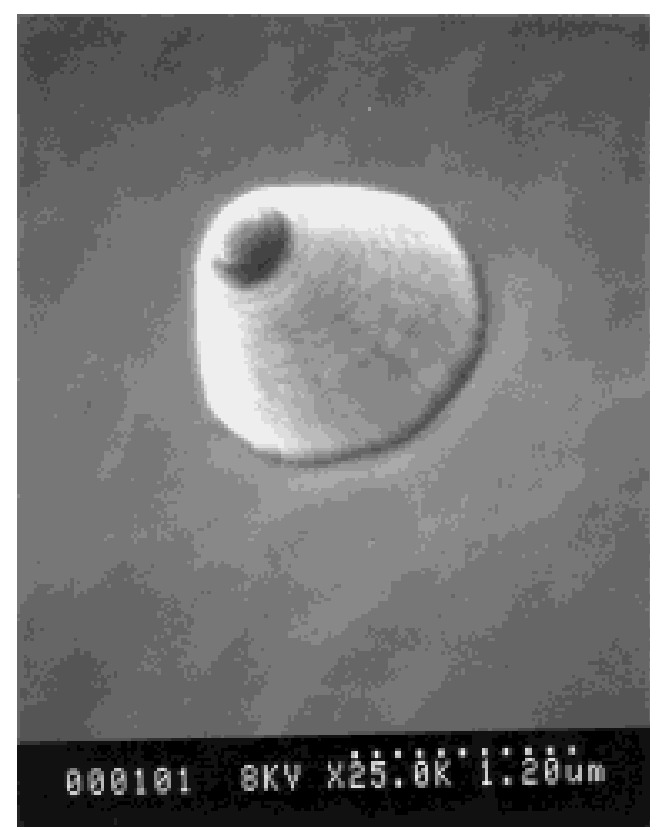

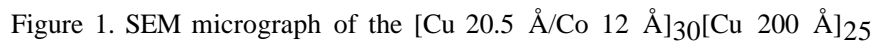
pedestal after the ECR etch. The base diameter is $1.5 \mu \mathrm{m}$ and the height is $1.1 \mu \mathrm{m}$.

film is deposited in situ to provide the conductive conduit to the sensor.

The next six process steps have been described elsewhere [3]. (1) Submicron diameter features are written using electron beam lithography. (2) The [Cu $20.5 \AA$ A $/$ Co $12 \AA]_{30}[\mathrm{Cu} 200$ $\AA]_{25}$ films are physically etched using an electron cyclotron resonance (ECR) etcher. (3) A $150 \mathrm{~nm}$ thick PECVD Si3 $\mathrm{N}_{4}$ film is deposited immediately following the ECR etch to passivate the metals. (4) The bottom Mo-Si ML contact is patterned using optical lithography, then reactive ion etched using the ECR plasma source. (5) A thick PECVD $\mathrm{Si}_{3} \mathrm{~N}_{4}$ film is deposited to provide the necessary electrical isolation between the bottom Mo-Si ML contact and the upper $\mathrm{Cu}$ contact. (6) The contact windows to the Mo-Si ML are patterned using optical lithography, then reactive ion etched using the ECR plasma source.

The SEM shown in Fig. 1 shows the [Cu $20.5 \AA$ A/Co 12 $\AA] 30[\mathrm{Cu} 200 \AA] 25$ stack after the ECR etch. The as-exposed features are nominally $0.46 \mu \mathrm{m}$ diameter pedestals. The postECR-etch features are conical with base diameters of approximately $1.5 \mu \mathrm{m}$. The pedestals are approximately 1.1 $\mu \mathrm{m}$ tall. The ECR etch used at this process step is primarily a physical etch. The expansion of the base width and the shape of the $\mathrm{Cu}$ conduit are caused by re-deposition of the 500 $\mathrm{nm}$ of $\mathrm{Cu}$. This problem can be minimized by replacing the $\mathrm{Cu}$ with a metal that can be reactive ion etched.

The $\mathrm{Cu}$ conduit is exposed by CMP. The PECVD $\mathrm{Si}_{3} \mathrm{~N}_{4}$ provides an excellent surface for "local planarization" by CMP. There are two characteristics of the CMP process that permit local planarization. First, CMP removes surface protrusions at a much greater rate than the removal rate of the surface. The time required to planarize the region occupied by each $[\mathrm{Cu} 20.5 \AA / \mathrm{Co} 12 \AA]_{30}[\mathrm{Cu} 200 \AA]_{25}$ stack, therefore, is independent of the removal rate of the surface. Second, the 


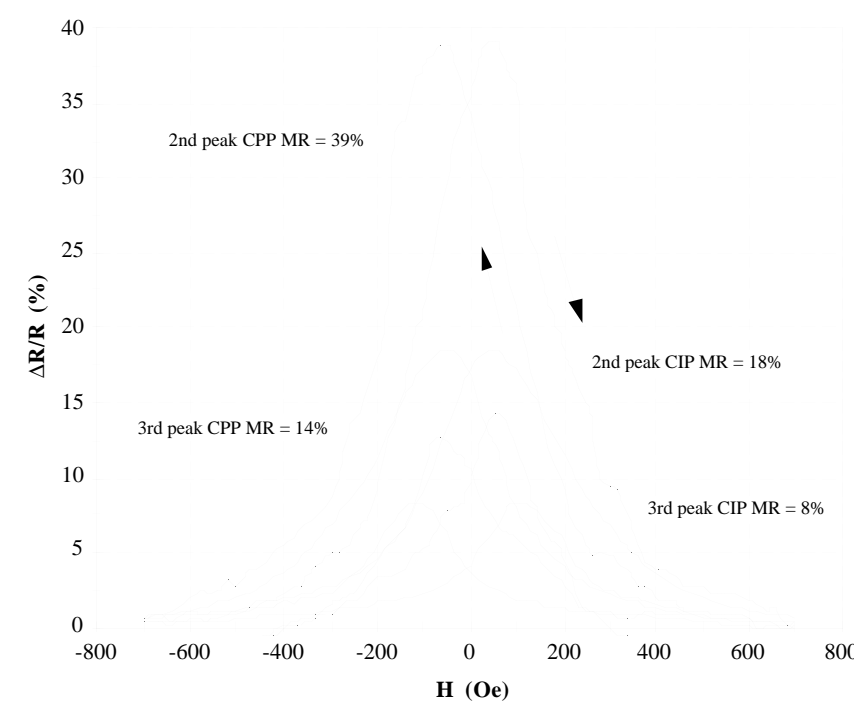

Figure 2. The second peak and third peak CPP and CIP GMR responses. The maximum calculated second peak CPP response is $39 \%$. The maximum calculated third peak CPP response is $14 \%$. The second peak CIP response is $18 \%$ and the third CIP peak response is $8 \%$.

CMP removal rate of PECVD $\mathrm{Si}_{3} \mathrm{~N}_{4}$ is typically much slower than the rate of PECVD $\mathrm{SiO}_{2}$. Using a PECVD $\mathrm{Si}_{3} \mathrm{~N}_{4}$ passivation film allows one to develop a CMP process that reduces the surface roughness and removes surface protrusions (i.e., locally planarizes) while removing less than $50 \mathrm{~nm}$ of the PECVD $\mathrm{Si}_{3} \mathrm{~N}_{4}$ film. Because the removal rate is low, the polishing can be continued as long as is required to planarize the region occupied by each $[\mathrm{Cu} 20.5 \AA$ $\AA]_{30}[\mathrm{Cu} 200 \AA]_{25}$ stack without excessive, non-uniform PECVD Si3 $\mathrm{N}_{4}$ film loss. The insensitivity to the endpoint is particularly important when etching large square $\mathrm{Al}_{2} \mathrm{O}_{3}-\mathrm{TiC}$ substrates because the etch rate is non-uniform at the substrate corners.

The $\mathrm{Cu}$ conduit is exposed by CMP if the combined thickness of the two PECVD $\mathrm{Si}_{3} \mathrm{~N}_{4}$ films is less than the

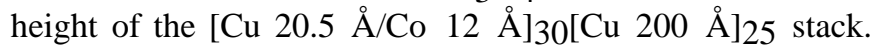
The final thickness of the PECVD $\mathrm{Si}_{3} \mathrm{~N}_{4}$ also determines the length of the $\mathrm{Cu}$ conduit. A $500 \mathrm{~nm} \mathrm{Cu}$ film is deposited following CMP and an Ar ion etch back. The top contact is patterned using optical lithography, then reactive ion etched using the ECR plasma source.

\section{QUASI-STATIC TESTING}

The QST station has been previously described [3]. Fig. 2 shows the CPP GMR response from a second and third peak test device and the CIP GMR response from a second and third peak as-deposited sheet film. The applied magnetic field is swept from -750 Oe to 750 Oe. The second peak CPP response is determined using the following parameters: $d=$ $1.5 \mu \mathrm{m}, \rho=18.5 \mu \Omega-\mathrm{cm}$, and $\mathrm{t}=97.5 \mathrm{~nm}$, where $\mathrm{d}$ is the sensor diameter, $\rho$ is the sheet resistivity of the ML, and $t$ is the thickness of the ML. The second peak CPP test current is $4 \mathrm{~mA} \mathrm{dc}$ with a $3 \mathrm{~mA}$ 0-to-peak sinusoidal modulation. The calculated second peak sensor resistance is $10.2 \mathrm{~m} \Omega$. The device resistance is $537 \mathrm{~m} \Omega$. The maximum calculated second 


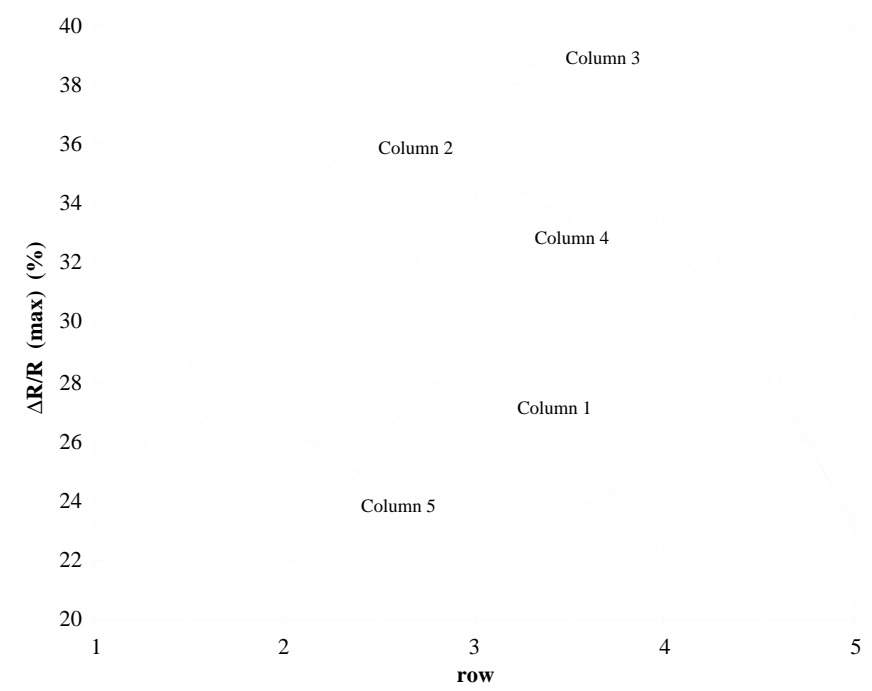

Figure 3. The second peak CPP GMR response of twenty devices from a single wafer. The response averaged over twenty devices is $28 \% \pm 6 \%$. The response averaged over the nine devices at the center of the wafer is $33 \% \pm 4 \%$

peak CPP response from a single device is $39 \%$. The saturation field is $300 \mathrm{Oe}$, the sensitivity is $0.13 \% / \mathrm{Oe}$, and the coercivity is 50 Oe. The third peak CPP response is determined using $\mathrm{d}=0.43 \mu \mathrm{m}, \rho=18.5 \mu \Omega-\mathrm{cm}$, and $\mathrm{t}=90.0$ $\mathrm{nm}$. The third peak CPP test current is $2 \mathrm{~mA}$ dc with a $1 \mathrm{~mA}$ 0 -to-peak sinusoidal modulation. The calculated third peak sensor resistance is $114.4 \mathrm{~m} \Omega$. The device resistance is 8.71 $\Omega$. The high device resistance is attributed to the $\mathrm{Au}$ electroplating process [3]. The maximum calculated third peak CPP response from a single device is $14 \%$. The saturation field is $250 \mathrm{Oe}$, the sensitivity is $0.07 \% / \mathrm{Oe}$, and the coercivity is $90 \mathrm{Oe}$. The maximum $\Delta \mathrm{V}$ is $8.4 \mu \mathrm{V}$ at $2.12 \mathrm{~mA}$ for the second peak device and $12.7 \mu \mathrm{V}$ at $0.71 \mathrm{~mA}$ for the third peak device.

The CIP GMR responses are determined by four-point probe using as-deposited sheet films. The current is $2 \mathrm{~mA} \mathrm{dc}$ with a $1 \mathrm{~mA}$ 0-to-peak sinusoidal modulation for both the second and third peak CIP tests. The second peak CIP response is $18 \%$ and the third peak response is $8 \%$. The second peak CIP response sensitivity is $0.08 \% / \mathrm{Oe}$ and the third peak CIP response sensitivity is $0.05 \% / \mathrm{Oe}$. The second peak and third peak CIP coercivities are 50 Oe and $110 \mathrm{Oe}$, respectively. The second peak and third peak CIP saturation fields are 255 Oe and 210 Oe, respectively. Note that the maximum second and third peak CPP responses are approximately twice the corresponding CIP responses. This result is consistent with published experimental $\mathrm{Cu}-\mathrm{Co} \mathrm{ML}$ response data [6]. The CPP and CIP responses are isotropic.

Fig. 3 shows the second peak CPP GMR response from twenty devices on a single wafer. The columns are perpendicular to the major flat. The devices are separated by $15 \mathrm{~mm}$. The shape of the second peak CPP response shown in Fig. 2 is representative of the shape of all twenty responses from the wafer. The average device resistance measured is $539 \mathrm{~m} \Omega \pm 12 \mathrm{~m} \Omega$. The CPP response averaged over twenty devices on a four inch silicon substrate is $28 \% \pm$ 
$6 \%$. The average sensitivity is $0.09 \% / \mathrm{Oe} \pm 0.02 \% / \mathrm{Oe}$ and the average coercivity is $47.4 \mathrm{Oe} \pm 8.0 \mathrm{Oe}$. The response decreases radially from the wafer center. The CPP response averaged over the nine devices at the center of the wafer is $33 \% \pm 4 \%$. The average center values for the sensitivity, saturation field, and coercivity are $0.11 \% / \mathrm{Oe} \pm 0.02 \% / \mathrm{Oe}$, $296.7 \mathrm{Oe} \pm 27.8 \mathrm{Oe}$, and $48.3 \mathrm{Oe} \pm 7.5 \mathrm{Oe}$, respectively.

\section{CONCLUSION}

We have fabricated and tested $\mathrm{Cu}-\mathrm{Co}$ GMR ML test devices designed for CPP mode operation. The following conclusions can be made based on the data: (1) The calculated second peak GMR response from a CPP mode device is as large as 39\%. (2) The CPP response is approximately twice the CIP response for both second and third peak ML spacings. (3) The response is isotropic in both the CPP and CIP modes. (4) The response is hysteretic. (5) The output voltage does not satisfy our specifications. (6) The sensitivity does not satisfy our specifications. The last three conclusions are problems that will need to be corrected to realize a practical magnetic flux sensor. We are currently addressing these problems. First, we are substituting the $\mathrm{Cu}$ conduit with a metal that can be reactive ion etched. This makes is possible to fabricate test devices with sub- $0.5 \mu \mathrm{m}$ diameters. Reducing the radius by a factor of three will cause a nine fold increase in $\Delta \mathrm{V}$. Further, a sensor composed of a single magnetic domain is expected to be more sensitive and less hysteretic than its multidomain counterpart. By fabricating deep submicron diameter sensors, we hope to realize a large increase in $\Delta \mathrm{V}$ and possibly benefit by single domain behavior. Second, we are fabricating devices using alternative GMR ML pairs and novel ML configurations.

\section{REFERENCES}

[1] R. Rottmayer and J. G. Zhu, "A new design for an ultra-high density magnetic recording head using a GMR sensor in the CPP mode," IEEE Trans. Magn., vol. 31(6), pp. 2597-2599, 1995.

[2] W. P. Pratt, et al., "Perpendicular Giant Magnetoresistances of Ag/Co mulitlayers," Phys. Rev. Lett., vol. 66(23), pp. 3060-3063, 1991.

[3] J. Spallas, et al., "Perpendicular current giant magnetoresistance in a $0.4 \mu \mathrm{m}$ diameter GMR Co-Cu multilayer sensor," IEEE Trans. Magn., vol. 32(5), pp. 4710-4712, 1996.

[4] D. G. Sterns, S. L. Baker, and M. A. Wall, "Ultrasmooth, conducting films composed of Mo/Si multilayers," Mat. Res. Soc. Symp. Proc., vol. 403, pp. 183-188, 1996.

[5] Y. Huai, S. P. Vernon, D. G. Stearns, C. Cerjan, and D. R. Kania, "Large GMR values of sputtered Co/Cu multilayer structures with CoCu buffer layers," presented at INTERMAG 97, Seattle, WA, 1996 (unpublished).

[6] M. A. M. Gijs, et al., "Perpendicular giant magnetoresistance of microstructures in $\mathrm{Fe} / \mathrm{Cr}$ and $\mathrm{Co} / \mathrm{Cu}$ multilayers," J. Appl. Phys., vol. 75(10), pp. 6709-6713, 1994. 


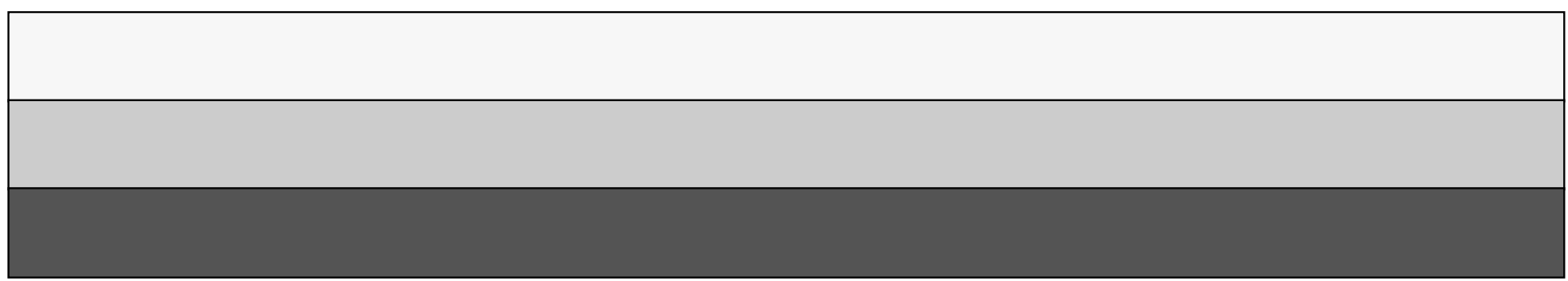

